\title{
Gum Arabic and Tricalcium Phosphate as Encapsulating Agents during kiwifruit Drying
}

\author{
M. Benlloch-Tinoco, M. Igual, M.M. Camacho, N. Martínez-Navarrete ${ }^{1 *}$ \\ Universitat Politècnica de València, Food Technology Department, Food Investigation and Innovation Group, Camino de Vera s/n, 46022 Valencia, Spain. \\ ${ }^{*}$ Corresponding author: N. Martínez-Navarrete, Universitat Politècnica de València, Food Technology Department, Food Investigation and Innovation Group, Camino \\ de Vera s/n, 46022 Valencia, Spain; Tel: +34 963879362; Fax: +34 96387 73 69; E-mail: nmartin@tal.upv.es
}

Received: November 27, 2019; Accepted: November 27, 2019; Published: December 15, 2019

\begin{abstract}
The current interest in the healthfulness of fruits may be linked to its antioxidant capacity, related to the presence of bioactive compounds. Freezedrying opens up new alternatives for fruit processing, mainly due to the scarce heat damage to the product. Nevertheless, the high cost of this process may recommend some pre-drying treatment. In this study, the effect that using pre-microwaves or hot air partial drying prior to freeze-drying has on the vitamin A, C and E content and antioxidant capacity of kiwifruit puree, with or without the presence of gum Arabic and tricalcium phosphate, was studied. Powders obtained from kiwifruit purees with solutes added showed a significant $(p<0.05)$ higher content of vitamins and greater antioxidant capacity. Gum Arabic and tricalcium phosphate protect against loss caused by any studied drying treatments. Nevertheless, microwaves and especially hot air partial drying treatments prior to freeze-drying caused significant vitamin and antioxidant capacity loss.
\end{abstract}

Keywords: microwaves; hot air; freeze-drying; antioxidant capacity; vitamins A, E and C

\section{Introduction}

Every day, our body is subjected to attack by many reactive oxygen species (ROS), formed not only during the physiological metabolism of energetic nutrients but also under infection, smoking, and pollution threaten $[1,2]$. When the normal antioxidant defences are overwhelmed, ROS bring about a condition of oxidative stress [3], which means that ROS attack and oxidize portions of lipids, nucleic acids, and proteins, with the consequent impairment of physiological functions and the appearance of pathologies [1]. The contribution of antioxidant nutrients becomes of vital importance to rescuing the body from oxidative stress conditions [4]. Fresh vegetables are relevant reservoirs of vitamins and phenol compounds [5]. If introduced into the body at every meal, those nutrients provide consistent protection to physiological macromolecules by increasing the antioxidant capacity of the plasma $[6,7]$. The kiwifruit is the edible berry of a cultivar group of the woody vines of several Actinidia species. The most common commercially available, green-fleshed kiwifruit is the 'Hayward' cultivar, which belongs to the Actinidia deliciosa species [8]. Kiwifruit are often promoted for their high vitamin $\mathrm{C}$ content, which probably contributes to the health benefits that include antioxidant, antiatherogenic and anticarcinogenic activities, as well as immunomodulation [9]. However, kiwifruit also contain other vitamins and minerals that may contribute to possible health benefits, including folate, potassium and magnesium, dietary fibre and phytochemicals [10]. The effects of kiwifruit consumption on hypertension and dyslipidemia have been tested in human trials. [11] found that the consumption of green kiwifruit resulted in a small but significant reduction in diastolic and systolic blood pressure in male smokers, the effect being strongest in those with hypertension.
On the other hand, in subjects that were hypercholesterolemic, kiwifruit consumption did have a favourable effect on plasma highdensity lipoprotein cholesterol (HDL-C) concentrations and the total cholesterol (TC): HDL-C ratio [12]. These results support the earlier findings that kiwifruit consumption increased HDL-C and decreased the TC: HDL-C ratio and also decreased the plasma triglyceride concentrations $[13,14]$.

In addition to the "mainstream" health targets commonly used to define the benefits from functional food, a small number of studies demonstrate more novel bioactive effects of kiwifruit, such as "natural" sleep aid or wound healing. The consumption of "Hayward" kiwifruit in the evening has been shown to improve sleep onset, duration, and efficiency in adults with self-reported sleep disturbances [15]. In addition, the application of kiwifruit fresh slices to burns has been shown to improve the healing process [16].

However, many people are not able to consume fresh fruit every day, due to their work conditions, distance from the markets of fresh products, or having very little time for shopping and cooking [17]. Therefore, they buy processed fruit and consume them every day. Powdered fruit products may be an alternative means of increasing fruit consumption in response to the increased demand for ready-toeat foodstuffs. The benefits of handling, packaging and transporting fruit powder, the great stability of the product and the ease of final consumption could contribute to this end. Freeze-drying (FD) emerged as a drying method which generates high quality products with very low moisture content, good sensory and nutritional properties and a good capacity for rehydration. However, long processing times and high operation costs are necessary to obtain freeze-dried products with an adequate level of quality (18). Different studies have been 
carried out into how to reduce processing costs, for example abolishing the cost of vacuum generation, combining technologies prior to or during FD operation or enhancing heat transfer [19-21]. On the other hand, freeze-dried fruit products are highly hygroscopic and are prone to suffering changes in their physical properties brought about by the environment and time. Therefore, adding biopolymers and other solutes has been shown to be necessary in order to increase the product stability by acting as a barrier to water adsorption [22, 23]. The aim of this study was to evaluate the impact of both the use of microwave (MW) or hot-air drying (HA) as pre-drying treatments prior to freeze-drying and the presence of gum Arabic (GA) and tricalcium phosphate (TCP) on both the vitamins and antioxidant capacity of kiwifruit powders.

\section{Materials and Methods}

\section{Sample preparation and treatments}

Kiwifruit (Actinidadeliciosa var. Hayward) was purchased in a local market in Valencia (Spain). The mean values (with standard deviation in brackets) of $\mathrm{pH}$, ${ }^{\circ} \mathrm{Brix}$ and water content of the kiwifruit used were 3.32 (0.09), $13.6(0.7)$ and $84.0(0.8) \mathrm{g} / 100 \mathrm{~g}$, respectively. The fruit pieces were peeled, washed with distilled water and triturated in a Thermomix (TM 21, Vorwerk, Spain). The obtained puree was divided into two parts and GA (Sigma CAS: 9000-01-5, Spain) along with TCP (Sigma CAS: 7758-87-04, Spain) were added to one of them. The quantities employed were: $1 \mathrm{~kg} \mathrm{GA} / \mathrm{kg}$ soluble solids of the liquid phase of the product [24] and $0.02 \mathrm{~kg} \mathrm{TCP} / \mathrm{kg}$ soluble solids of the liquid phase of the product $[25,26]$. As a result, two different samples were obtained: kiwifruit and kiwifruit with solutes. A part of each sample was freeze-dried. To this end, a layer ( $5 \mathrm{~mm}$ thickness) of each sample was placed in a standardised aluminium plate $(15 \mathrm{~cm}$ diameter and $5 \mathrm{~cm}$ height). Consecutively, samples were stored in a CVF 525/86 cryo freezer $\left(-86^{\circ} \mathrm{C}\right)$ (Ing. Climas, Spain) for the $24 \mathrm{~h}$ before being freeze-dried in a Lioalfa-6 Lyophyliser (Telstar, Spain) at 0.026 mbar and $-56.6^{\circ} \mathrm{Cat}$ condenser for $24 \mathrm{~h}$. The obtained cakes were crushed (Thermomix TM 21, Vorwerk, Spain) to obtain kiwifruit powders, with and without solutes, which were named KS and K, respectively.

The rest of the samples of kiwifruit and kiwifruit with solutes was submitted to two different pre-drying methods, prior to FD, in order to reduce the initial water content: MW and HAD. According to preliminary experiments (data not shown), the final water content for pre-treatments was set at $65 \mathrm{~g}$ water/100 $\mathrm{g}$ sample. This moisture level led to a sample of good appearance with noticeable water loss, without the need for an excessively long pre-treatment time. To ensure this water content in the samples, their weight was recorded during the process. It allowed for the calculation of the water content at different drying times, provided the initial water content is known. Puree samples of kiwifruit and kiwifruit with solutes $(300 \mathrm{~g})$ were placed in standard recipients $(25 \mathrm{~cm}$ diameter and $3 \mathrm{~cm}$ depth) made of Teflon (Mecaplast, Spain) or aluminium for MW and HAD, respectively. A household microwave (3038GC, Norm, China), set at a microwave power of $2 \mathrm{~W} / \mathrm{g}$ and an oven (5141 AFW2, Moulinex, China), set at an air temperature of $40{ }^{\circ} \mathrm{C}$ and an air velocity of $1.5 \mathrm{~m} / \mathrm{s}$, were used to carry out the drying pre-treatments. Microwave power and air temperature were selected by taking into account the minimal loss of antioxidant capacity obtained in preliminary experiments (data not shown). The treated samples were immediately cooled in ice water until the puree reached $30{ }^{\circ} \mathrm{C}$. All four samples were freeze-dried as described above to obtain the corresponding powders, thus becoming freeze-dried kiwifruit puree with and without solutes added, predried by MW or HAD, named as follows: KMW, KHAD, KSMW and KSHAD, respectively.

\section{Analytical determinations}

All the analyses described below were carried out in triplicate on fresh and freeze-dried kiwifruit samples. Despite the obtained results being referred to $100 \mathrm{~g}$ of each sample, the losses of vitamins, total phenolic compounds and antioxidant capacity suffered by the samples were calculated and also referred to $100 \mathrm{~g}$ of the kiwifruit's own solutes (KS) in order to compare them(equations 1 and 2).

$$
\begin{aligned}
& x_{w}=\frac{x_{w}^{p}}{\left(1-x_{w}^{p}\right)\left(x_{G S / T S}\right)} \\
& x_{G S / T S}=\frac{m_{L}\left(1-x_{w}^{L}\right)}{m_{G A}+m_{C M C} \pm m_{L}\left(1-x_{w}^{L}\right)}
\end{aligned}
$$

where: $x_{w}{ }^{p}$ is the water content of the powder $(\mathrm{w} / \mathrm{w}), x_{G S / T S}$ is the mass fraction of the grapefruit's own solutes (GS) to total sample solutes, $m_{G A}, m_{C M C}$ and $m_{L}$ are the mass of gum Arabic, carboxymethyl cellulose and liquidized grapefruit, respectively, in the sample and $x_{w}{ }^{L}$ is the water content of the liquidized grapefruit (w/w).

The mass fraction of water was obtained by vacuum drying the samples in a vacuum oven (Vaciotem, J.P. Selecta, Spain) at 60 $\pm 1{ }^{\circ} \mathrm{C}$ under a pressure of $<100 \mathrm{~mm} \mathrm{Hg}$ until constant weight. To determine the total solute content (TS) of the freeze-dried samples, the methodology described by [27] was followed. In turn, $1.5 \mathrm{~g}$ of each powder were rehydrated, in triplicate, and the TS of the bulk suspension were determined by oven drying (Vaciotem, J.P. Selecta, Spain) at $60^{\circ} \mathrm{C}$ until constant weight.

\section{Vitamins $\mathrm{A}, \mathrm{C}$ and $\mathrm{E}$}

The vitamins were determined by HPLC (Jasco equipment, Italy). The procedure employed to determine vitamin $\mathrm{C}$ was the reduction of dehydroascorbic acid to ascorbic acid, using DL-dithiothreitol as reductant reagent according to Igual et al., 2016. The HPLC method and instrumentation were: Ultrabase-C18, $5 \mu \mathrm{m}(4.6 \times 250 \mathrm{~mm})$ column (AnálisisVínicos, Spain); mobile phase $0.1 \%$ oxalic acid, volume injection $20 \mu \mathrm{L}$, flow rate $1 \mathrm{~mL} / \mathrm{min}$, detection at $243 \mathrm{~nm}$ and at $25^{\circ} \mathrm{C}$. AA standard solution (Panreac, Spain) was prepared.

Vitamins A and E were extracted twice in the hexane phase and the collected extract was dried under a stream of liquid nitrogen [28]. The dried extract was solubilized in $0.2 \mathrm{~mL}$ methanol. The HPLC method and instrumentation were: Ultrabase-C18, $5 \mu \mathrm{m}(4.6$ $\times 250 \mathrm{~mm}$ ) column (Spain); mobile phase methanol/acetonitrile/ chloroform (47:42:11, v/v/v), volume injection $20 \mu \mathrm{L}$, flow rate $1 \mathrm{~mL} /$ min, detection at 326 and 296 for vitamins A and E, respectively at 25 ${ }^{\circ} \mathrm{C}$. Standard curves of each reference compound (Fluka-Biochemika, USA) were used for quantification purposes. 


\section{Antioxidant capacity}

Antioxidant capacity (AOC) was assessed using the free radical scavenging activity of the samples evaluated with the stable radical 2, 2-diphenyl-1-picryl-hydrazyl-hydrate (DPPH). Briefly, the samples were diluted in methanol, homogenized and centrifuged (Selecta Medifriger-BL, Spain) at $400 \mathrm{x} \mathrm{g}$ for $10 \mathrm{~min}$ at $4{ }^{\circ} \mathrm{C} .0 .1 \mathrm{~mL}$ of supernatant was added to $3.9 \mathrm{~mL}$ of DPPH (Sigma-Aldrich, Germany) diluted in methanol $(0.030 \mathrm{~g} / \mathrm{L})$. At $30 \mathrm{~s}$ intervals, a UV-visible spectrophotometer was used to measure the absorbance at $25{ }^{\circ} \mathrm{C}$ and515 nm until the reaction reached the steady state. The percentage of DPPH was calculated following Eq. (3).

$$
\% \mathrm{DPPH}=\frac{\left(\mathrm{A}_{\text {control }}-A_{\text {sample }}\right)}{\mathrm{A}_{\text {control }}} 100
$$

where $\mathrm{A}_{\text {control }}$ is the absorbance of the control (initial time) and $\mathrm{A}_{\text {sample }}$ the absorbance of the sample at the steady state.

The final results were expressed as mmol trolox equivalents (TE) per $100 \mathrm{~g}$ sample using a trolox calibration curve in the range of 6.25$150 \mathrm{mM}$ (Sigma-Aldrich, Germany).

\section{Statistical analysis}

An analysis of variance (ANOVA) was carried out to evaluate the differences among samples. When the $\mathrm{p}$ value was lower than 0.05 , significant differences between samples were assumed. Furthermore, an analysis of the correlation between AOC and all the studied vitamins, with a $95 \%$ significance level, was carried out. All statistical analyses were performed using Stat graphics Centurion XVI.II for Windows.

\section{Results and discussion}

\section{Kiwifruit characterization}

The vitamin A content $(0.029(0.002) \mathrm{mg} / 100 \mathrm{~g})$ was lower than that of the rest of the quantified bioactive compounds. The vitamin $\mathrm{E}$ content was $2.10(0.13) \mathrm{mg} / 100 \mathrm{~g}$, similar to the values of this vitamin shown by [29] (2.5 mg /100g,). In general, citric fruit are considered a good source of vitamin C. However, the amount of vitamin C in kiwifruit $(105(5) \mathrm{mg} / 100 \mathrm{~g})$ is about three times more than that present in $100 \mathrm{~g}$ of grapefruit [30] or twice more than orange [31]. The obtained results coincide with the values shown for kiwifruit var. Hayward by other authors [32-35]. One of the predominant mechanisms of the protective action of bioactive compounds is their antioxidant activity and the capacity to scavenge free radicals [36]. Kiwifruit showed $15.63(0.15) \mathrm{mmol}$ TE/100g of AOC.

The effect of solute addition and pre-drying on bioactive compound variation of freeze-dried kiwifruit powder

The losses of vitamins and AOC suffered by the samples as a consequence of each drying step have been plotted in Fig. $1-4$. The loss of each component $(\Delta \mathrm{Mi})$, referred to the fresh kiwifruit content, were calculated according to equations 4 and 5 .

$$
\begin{gathered}
\Delta M_{i}^{P}=\frac{\left(M_{i}^{F K}-M_{i}^{P}\right)}{M_{i}^{F K}} \\
\Delta M^{F D}=\frac{\left(M_{i}^{P}-M_{i}^{F D}\right)}{M_{i}^{F K}}
\end{gathered}
$$

where: $M_{i}$ : mass of compound $i$ in the sample referred to the kiwifruit's own solutes and superscripts: FK: fresh kiwifruit, P: pretreated (mixed with the solutes and/or pre-dried by MW or HAD), FD: freeze-dried.

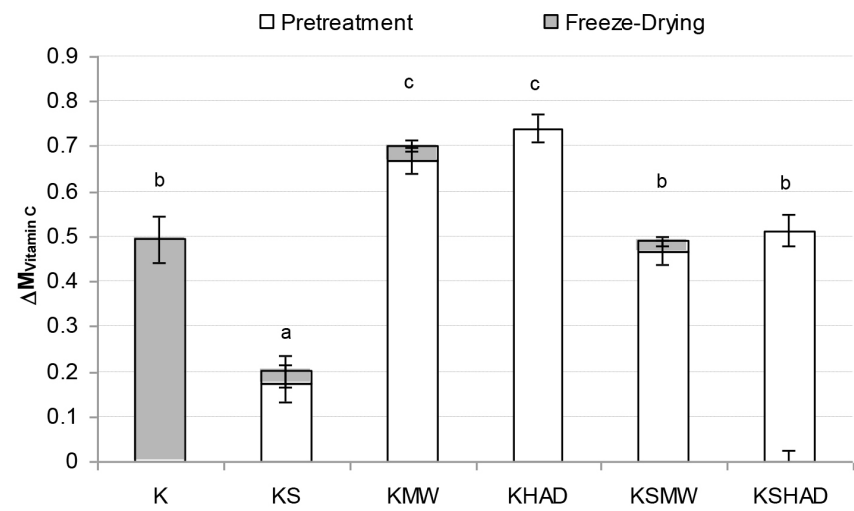

Figure 1. Mean value and standard deviation of Vitamin C losses suffered by the samples as a consequence of each process studied.

Letters indicate homogeneous groups established by the ANOVA $(p<0.05)$ for total process variation.

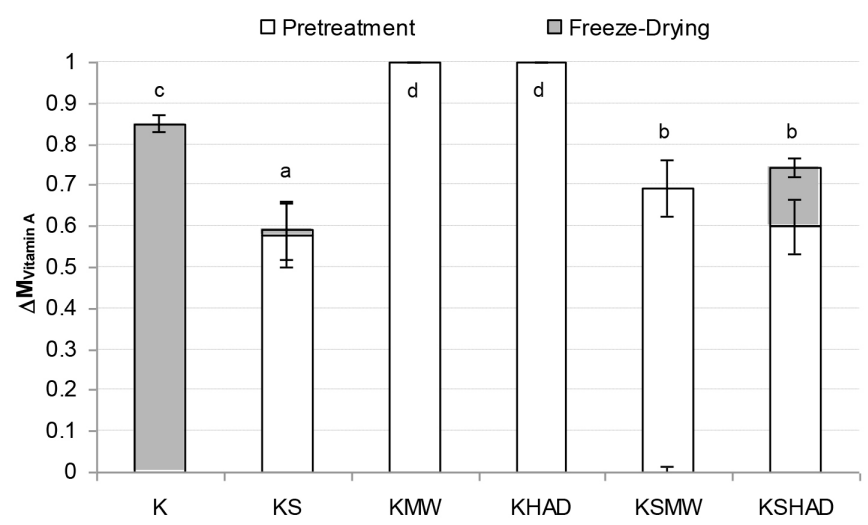

Figure 2. Mean value and standard deviation of Vitamin A losses suffered by the samples as a consequence of each process studied

Letters indicate homogeneous groups established by the ANOVA $(p<0.05)$ for total process variation.

As regards the vitamins studied (Figures $1-3$ ), FD was observed to bring about the greatest vitamin loss in sample $\mathrm{K}$. When comparing samples $\mathrm{K}$ and $\mathrm{KS}$, a loss of all three of the vitamins studied was observed due to the mixing process when solutes were added to the kiwifruit puree. Nevertheless, solutes reduced the losses caused by FD. In general, the application of MW or HAD pre-treatment led to an increase in vitamin losses, especially when no solutes were present. In this way, GA+TCP can be confirmed to exert a protective effect on the 
vitamins studied throughout FD.As regards pre-drying treatments, the loss of vitamins A or C due to MW or HAD pre-treatments was of the same order, while the loss of vitamin $\mathrm{E}$ was greater when $\mathrm{HAD}$ was used. In fact both the KMW and KHAD samples even lost the entire amount of vitamin A present in the fresh kiwi. In these KMW and KHAD samples, the additional loss of the remaining vitamin $\mathrm{C}$ brought about by the freeze-drying process was almost negligible, while the loss of vitamin $\mathrm{E}$ was also promoted in sample KMW. When comparing these samples with KSMW and KSHAD, the loss of vitamins was reduced in the samples with solutes added, so that for vitamins $\mathrm{A}$ and $\mathrm{C}$ the loss was reduced in the range of 30 and $20 \%$, respectively and for vitamin $\mathrm{E}$ from $15 \%$ to $40 \%$, depending on whether the HAD or the MW pre-treatment was applied, respectively. As regards AOC (Fig. 4), a loss of about $5 \%$ was observed caused by FD (sample K) or simply by sample formulation (sample KS). A 50-60\% loss of AOC was observed in pre-treated KMW and KHAD samples, due just to FD or to HAD and FD, respectively. This loss was reduced to 20-25 $\%$ when solutes were present (KSMW and KSHAD samples). Added solutes help the retention of nutritive properties of kiwifruit during pre-treatments and FD. Maltodextrins and gums are added during the production of food powders in order to act as encapsulating or wall materials, contributing to keeping the desired functional properties in the finished product, such as stability against oxidation, ease of handling, improved solubility, controlled release and extended shelflife [37]. GA is the gum most often used as a flavour encapsulating material, mainly due to its solubility, low viscosity, emulsification characteristics and its good retention of volatile compounds [38-40].

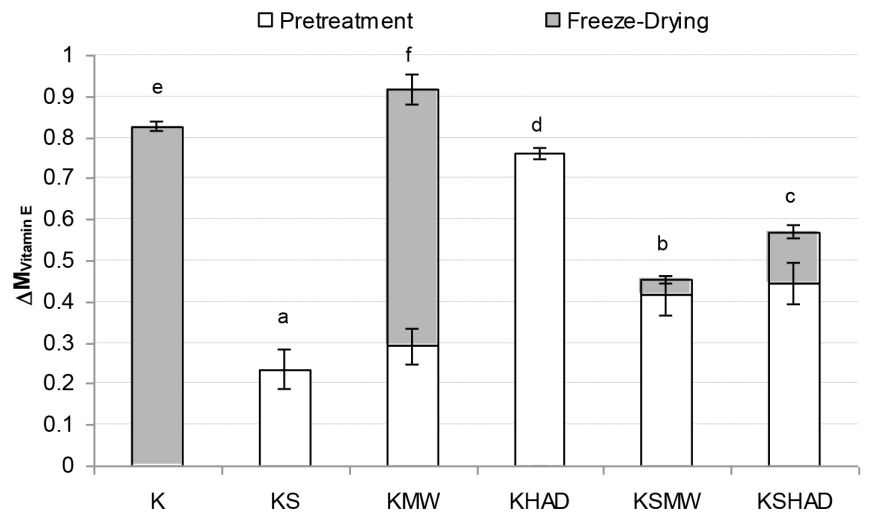

Figure 3. Measn value and standard deviation of Vitamin E losses suffered by the samples as a consequence of each process studied

Letters indicate homogeneous groups established by the ANOVA $(\mathrm{p}<0.05)$ for total process variation

Table 1 shows the mean value of vitamins and AOC contained in $15 \mathrm{~g}$ of the different obtained kiwifruit powders. About $15 \mathrm{~g}$ of kiwifruit powder is the equivalent to a fresh kiwi-fruit $(\approx 85 \mathrm{~g})$. The highest vitamin intake is provided by sample KS, despite the highest AOA being supplied by sample K. An intake of $15 \mathrm{~g}$ of $\mathrm{K}$ or KS powders or $\approx$ $23 \mathrm{~g}$ of any of the other powders is enough to meet the daily amount of vitamin $\mathrm{C}$ recommended by the World Health Organization $(\approx 40 \mathrm{mg})$. KMW, KHAD and KSHAD samples also showed the lowest AOC. In order to explain the relationship among the different vitamins quantified in this study themselves and also between them and the
AOC of the samples, correlation statistical analyses were performed using all the obtained data (Table 2). A significant relationship ( $\mathrm{p}>0.05$ ) between the AOC and any of the studied vitamins was observed, as was a closer relationship among the vitamins.

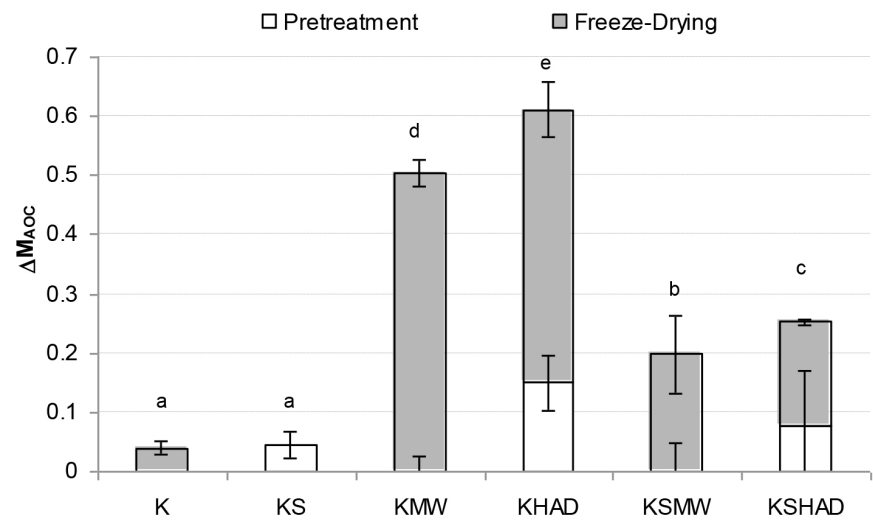

Figure 4. Mean value and standard deviation of antioxidant capacity losses suffered by the samples as a consequence of each process studied.

Letters indicate homogeneous groups established by the ANOVA $(p<0.05)$ for total process variation.

Table 1. Mean values (with standard deviation) of vitamins $(\mathrm{mg} / 15 \mathrm{~g}$ ) and antioxidant capacity (AOC) (mmolTE/15 g) of obtained powders: K (kiwifruit powders without solutes), KS (kiwifruit powders with solutes), KMW (kiwifruit powder without solutes added pre-dried by microwaves), KHAD (kiwifruit powder without solutes added predried by hot air), KSMW (kiwifruit powder with solutes added pre-dried by microwaves) and KSHAD (kiwifruit powder with solutes added pre-dried by hot air).

\begin{tabular}{|c|c|c|c|c|}
\hline Sample & Vitamin A & Vitamin C & Vitamin E & AOC \\
\hline K & $0,004(0,0005)^{\mathrm{bc}}$ & $43(5)^{\mathrm{a}}$ & $0.2990(0,0012)^{\mathrm{d}}$ & $12.24(0,13)^{\mathrm{a}}$ \\
\hline KS & $0,006(0,0003)^{\mathrm{a}}$ & $40.5(1.7)^{\mathrm{a}}$ & $0.813(0,012)^{\mathrm{a}}$ & $7.55(0,07)^{\mathrm{b}}$ \\
\hline KMW & $0^{\mathrm{d}}$ & $27.5(0.8)^{\mathrm{b}}$ & $0.184(0,015)^{\mathrm{c}}$ & $6.86(0,03)^{\mathrm{bc}}$ \\
\hline KHAD & $0^{\mathrm{d}}$ & $27.7(0.9)^{\mathrm{b}}$ & $0.473(0,012)^{\mathrm{c}}$ & $5.5(0.4)^{\mathrm{d}}$ \\
\hline KSMW & $0,004(0,0004)^{\mathrm{b}}$ & $26.8(0.7)^{\mathrm{b}}$ & $0.543(0,004)^{\mathrm{b}}$ & $6.05(0,06)^{\mathrm{cd}}$ \\
\hline KSHAD & $0,003(0,0002)^{\mathrm{c}}$ & $25.3(0.6)^{\mathrm{b}}$ & $0.470(0,003)^{\mathrm{c}}$ & $\begin{array}{c}5.5 \\
(60.9)^{\mathrm{d}}\end{array}$ \\
\hline
\end{tabular}

The same letter in superscript within columns indicates homogeneous group established by ANOVA $(\mathrm{p}<0.05)$.

Table 2. Pearson correlation coefficients among all studied components

\begin{tabular}{|l|c|c|c|}
\hline & Vitamin C & Vitamin A & Vitamin E \\
\hline AOC & $0,5787^{*}$ & $0,4728^{*}$ & $0,6143^{*}$ \\
\hline Vitamin C & & $0,7926^{*}$ & $0,8077^{*}$ \\
\hline Vitamin A & & & $0,846^{*}$ \\
\hline
\end{tabular}

* Correlation is significant at the 0.05 level.

\section{Conclusions}

An encapsulating effect of GA+TCP was observed both for the mixing with solutes and/or the pre-drying treatment and for the freezedrying process. When solutes are present, a pre-drying treatment 
with MW may be recommended instead of HAD for the purposes of obtaining a freeze-dried product, as vitamin $\mathrm{E}$ and AOC are better preserved. Nevertheless, despite the fact that the pre-treatment will shorten the subsequent freeze-drying time and so reduce the energy costs, if the maximum preservation of the vitamin content and AOC is desired, the use of microwave or hot air drying pre-treatment should be avoided.

\section{Acknowledgments}

The authors thank the Ministerio de Economía y Competitividad for the financial support given through the Project AGL 2017-89251R (AEI/FEDER-UE).

\section{References}

1. Dean RT, Fu S, Stocker R, Davies MJ (1997) Biochemistry and pathology of radical- mediated protein oxidation. Biochem J 324: 1-18. [crossref]

2. Finkel T, Holbrook NJ (2000) Oxidants, oxidative stress and the biology of aging. Nature 408: 239-247. [crossref]

3. Halliwell B, Cross CE (1994) Oxygen-derived species: Their relation to human disease and environmental stress. Environ Health Perspect 102: 5-12. [crossref]

4. Sun J, Chu YF, Wu X, Liu RH (2002) Antioxidant and Antiproliferative Activities of Common Fruits. J Agric Food Chem 50: 7449-7454. [crossref]

5. Rickman, J.C., Barret,D.M. \& Bruhn, C.M (2007) Review: Nutritional comparison of fresh and frozen fruits. Journal of Science and Food Agriculture 87: 930-944.

6. Ninfali P, Mea G, Giorgini S, Rocchi M, Bacchiocca M (2005) Antioxidant capacity of vegetables, spices and dressings relevant to nutrition. British J Nutr 93: 257-266. [crossref]

7. Prior RL, Gu L, Wu X, Jacob RA, Sotoudeh G et al. (2007) Plasma antioxidant capacity changes following a meal as a measure of the ability of a food to alter in vivo antioxidant status. J American Coll Nutr 26: 170-81. [crossref]

8. Fiorentino A, D'Abrosca B, Pacifico S, Mastellone C, Scognamiglio M et al. (2009) Identification and assessment of antioxidant capacity of phytochemicals from kiwi fruits. J Agric Food Chem 57: 4148-4155. [crossref]

9. Vissers MC, Carr AC, Pullar JM, Bozonet SM (2013) The bioavailability of vitamin C from kiwifruit. Adv Food Nutr Res 68: 25-147. [crossref]

10. Drummond L (2013) The Composition and Nutritional Value of Kiwifruit. Adv Food Nutr Res 68: 33-57. [crossref]

11. Karlsen A, Svendsen M, Seljeflot I, Laake P, Duttaroy AK et al. (2013) Kiwifruit decreases blood pressure and whole-blood platelet aggregation in male smokers. $J$ Human Hypertension 27: 126-130. [crossref]

12. Gammon C, Kruger R, Minihane A, Conlon C, Von Hurst P et al. (2013) Kiwifruit consumption favourably affects plasma lipids in a randomised controlled trial in hypercholesterolaemic men. British J Nutr 109: 2208-2218. [crossref]

13. Brevik A, Gaivão I, Medin T, Jørgenesen A, Piasek A et al. (2011) Supplementation of a western diet with golden kiwifruits (Actinidia chinensis var.'Hort 16A':) effects on biomarkers of oxidation damage and antioxidant protection. Nutr $J 10$ : 54. [crossref]

14. Duttaroy AK, Jøorgensen A (2004) Effects of kiwifruits consumption in human volunteers on platelet aggregation and plasma lipids in vitro. Platelets 15: 287-292. [crossref]

15. Lin HH, Tsai PS, Fang SC, Liu JF (2011) Effect of kiwifruit consumption on sleep quality in adults with sleep problems. Asia Pacific J Clinical Nutr 20: 169-174. [crossref]

16. Kooshiar H, Abbaspour H, Motamed Al Shariati SM, Rakhshandeh H, Khajavi Rad A et al. (2012) Topical effectiveness of kiwifruit versus fibrinolysin ointment on removal of necrotic tissue of full-thickness burns in male rats. Dermatol Therapy 25: 621-625. [crossref]

17. Bertuccioli A, Ninfali P (2014) The Mediterranean Diet in the era of globalization: The need to support knowledge of healthy dietary factors in the new socioeconomical framework. Mediterranean J NutrMetab 7(1): 75-86.

18. Menlik T, Özdemir MB, Kirmaci V (2010) Determination of freeze-drying behaviours of apples by artificial neural network. Expert Sys Appl 37: 7669-7677.

19. Zhang M, Tang J, Mujumdarc AS, Wang, S (2006) Trends in microwave related drying of fruits and vegetables. Trends Food Sci Technol 17: 524-534.

20. Pardo JM, Leiva DA (2010) Effects of different pre-treatments on energy consumption during freeze drying of pineapple pieces. Interciencia 35: 934-938.

21. Huang L, Zhang, Mujumdar AS, Lim R (2011) Comparison of four drying methods for re-structured mixed potato with apple chips. J Food Eng 103: 279-284.
22. Cozic C, Picton L, Garda M, Marlhoux F, Le Cerf D (2009) Analysis of arabic gum: study of degradation and water desorption processes. Food Hydrocoll 23: 1930-1934.

23. Telis VN, Martínez-Navarrete N (2012) Biopolymers used as drying aids in spray drying and freeze drying of fruit juices and pulps. In V. Telis (Ed.). Biopolymer Engineering in Food Processing (pp. 279-326).London: CRC Press. Taylor \& Francis Group.

24. Mosquera LH, Moraga G, Martínez-Navarrete N (2012) Critical water activity and critical water content of freeze-dried strawberry powder as affected by maltodextrin and arabic gum. Food Res Inter 47: 201-206.

25. Cubero N, Monferrer A, Villalta J (2002) Aditivos alimentarios. Madrid: A. Madrid Vicente.

26. Jaya, S., \& Das, H. (2009). Glass transition and sticky point temperatures and stability/mobility diagram of fruit powders. Food Bioprocess Technology 2: 89-95.

27. Benlloch-Tinoco M, Varela P, Salvador A, Martínez-Navarrete N (2012) Effects of microwave heating on sensory characteristics of kiwifruit puree. Food Bioprocess Technol 5: 3021-3031.

28. Igual M, García-Martínez E, Camacho MM, Martínez-Navarrete N (2016) Stability of micronutrients and phytochemicals of grapefruit jam as affected by the obtention process. Food Sci Technol Inter 22: 203-212. [crossref]

29. Rush EC, Patel M, Plank LD, Ferguson LR (2002) Kiwifruit promotes laxation in the elderly. Asia Pacific J Clin Nutr 11: 164-168. [crossref]

30. Igual M, García-Martínez E, Camacho MM, Martínez-Navarrete N (2010) Effect of thermal treatment and storage on the stability of organic acids and the functional value of grapefruit juice. Food Chem 118: 291-299.

31. Sánchez-Moreno C, Plaza L, De Ancos B, Cano MP (2003) Quantitative bioactive compounds assessment and their relative contribution to the antioxidant capacity of commercial orange juices. J SciFood Agric 83: 430-439.

32. De Ancos B, Cano MP, Hernández A, Monreal M (1999) Effects of microwave heating on pigment composition and color of fruit purees. J Sci Food Agric 79: 663-670.

33. Schwartz M, Nuñez H, Muñoz AM (1999) Effect of concentration temperature of kiwifruit pulp on colour, chlorophyll and ascorbic acid. Archivos Latino Nutr 49: 44-48. [crossref]

34. Antunes MDC, Dandlen S, Cavaco AM, Miguel G (2010) Effects of postharvest application of 1-mep and postcutting dip treatment on the quality and nutritional properties of fresh-cut kiwifruit. J Agric Food Chem 58: 6173-6181. [crossref]

35. Zolfaghari M, Sahari A, Barzegar M, Samadloiy H (2010) Physicochemical and enzymatic properties of five kiwifruit cultivars during cold storage. Food Bioprocess Technol 3: 239-246.

36. Yahia EM, Maldonado E, Svendsen M (2010) The contribution of fruit and vegetable consumption to human health. In: Yahia EM (Ed). Fruit and vegetable phytochemicals.(pp. 3-51), Wiley-Blackwell, USA.

37. Jafari SM, Mahdavi-Khazaei K, Hemmati-Kakhki A (2016) Microencapsulation of saffron petal anthocyanins with cress seed gum compared with Arabic gum through freeze drying. Carbohydrate Polymers 140: 20-25.

38. Madene A, Jacquot M, Scher J, Desobry S (2006) Flavour encapsulation and controlled release - a review. Inter J Food Sci Technol 41: 1-21.

39. Righetto AM, Netto FM (2006) Vitamin C stability in encapsulated green West Indian cherry juice and in encapsulated synthetic ascorbic acid. J Sci Food Agric 86: $1202-1208$.

40. Desobry SA, Netto FM, Labuza TP (1997) Comparison of spray-drying, drumdrying and freeze-drying for $\beta$-carotene encapsulation and preservation. $J$ Food Sci 62: $1158-1162$.

\section{Citation:}

M. Benlloch-Tinoco, M. Igual, M.M. Camacho (2019) Gum Arabic and Tricalcium Phosphate as Encapsulating Agents during kiwifruit Drying. Nutr Diet Nutraceuticals 1(1): 1-5. 